\title{
Determination and Pharmacokinetic Study of Dauricine in Rat Plasma by UPLC-MS/MS
}

\author{
Peiwu Geng ${ }^{1}$, Jing Zhang ${ }^{2}$, Bingbao Chen ${ }^{2}$, Qianqian Wang ${ }^{2}$, Shuanghu Wang ${ }^{1}$ and Congcong Wen ${ }^{2 *}$ \\ ${ }^{1}$ The Laboratory of Clinical Pharmacy, The People's Hospital of Lishui, Wenzhou Medical University, Lishui 323000, China \\ ${ }^{2}$ Laboratory Animal Centre, Wenzhou Medical University, Wenzhou 325035, China
}

Received: 11 September 2016; accepted: 06 October 2016

\begin{abstract}
Dauricine is the major bioactive component isolated from the roots of Menispermum dauricum D.C., a bisbenzylisoquinoline alkaloid derivative, and has shown multiple pharmacological properties. In this work, a sensitive and selective ultra-performance liquid chromatography-tandem mass spectrometry (UPLC-MS/MS) method was developed for determination of dauricine in rat plasma and its application to pharmacokinetic study of dauricine after intravenous and oral administration in rats. After addition of daurisoline as an internal standard (IS), protein precipitation by acetonitrile was used to prepare samples. Chromatographic separation was achieved on a UPLC BEH C18 column $(2.1 \mathrm{~mm} \times 50 \mathrm{~mm}, 1.7 \mu \mathrm{m})$ with $0.1 \%$ formic acid and acetonitrile as the mobile phase with gradient elution. An electrospray ionization source was applied and operated in positive ion mode; multiple reactions monitoring (MRM) mode was used for quantification. Calibration plots were linear throughout the range 2-600 ng $\mathrm{mL}^{-1}$ for dauricine in rat plasma. Relative standard deviation (RSD) of intra-day and inter-day precision was less than $13 \%$. The accuracy of the method was between $95.8 \%$ and $105.9 \%$. Matrix effect of dauricine in rat plasma ranged from $88.0 \%$ to $90.3 \%$. Mean recoveries of dauricine in rat plasma ranged from $91.5 \%$ to $95.1 \%$. The method was successfully applied to pharmacokinetic study of dauricine after intravenous and oral administration in rats. The bioavailability of dauricine was found to be $55.4 \%$ for the first time.
\end{abstract}

Keywords: Dauricine, UPLC-MS/MS, pharmacokinetics, rat, plasma

\section{Introduction}

Dauricine (Figure 1) is a bisbenzylisoquinoline alkaloid isolated from the root of Menispermum dauricum D.C. The herb is often included in remedies for the treatment of antiplatelet aggregation, throat swelling, and chronic bronchitis $[1,2]$. Dauricine has been suggested for the treatment of various diseases, including cardiac ischemia, angina, and inflammation [3-5]. The alkaloid has shown varieties of bioactive properties, including antiarrhythmic, antitumor, anti-inflammatory, and neuron-protective effects. Thus, dauricine has attracted substantial attention due to its multiple pharmacologic activities and abundance in natural source [6, 7]. Therefore, it is necessary to develop a sensitive and fast bioanalytical method to characterize the pharmacokinetics.

There was a liquid chromatography method developed for the quantification of dauricine in beagle dog plasma to characterize the pharmacokinetics [8]. There, a sensitive and selective liquid chromatography-tandem mass spectrometry method had been developed and validated for simultaneous quantitation of 10 alkaloids (daurisoline, dauricine, $\mathrm{N}$-desmethyl daurisoline, dauricicoline, dauriporphinoline, bianfugecine, dauricoside, stepholidine, acutumine, and acutumidine) from Rhizoma Menispermi in rat plasma, and the validated method was successfully applied to a comparative pharmacokinetic study of 10 alkaloids in rat plasma after oral administration of Rhizoma Menispermi extract [9]. Liu et al. developed a liquid chromatography-tandem mass spectrometry method for quantitative determination of dauricine in human plasma and its application to pharmacokinetic study, with liquid-liquid extraction for sample preparation [10]. However, the bioavailability of dauricine was not reported in these literatures.

In comparison with conventional analytical techniques, ultraperformance liquid chromatography coupled with tandem mass spectrometry (UPLC-MS/MS) is documented to possess im-

* Author for correspondence: bluce494949@163.com proved sensitivity, selectivity, and specificity in quantitative determination of the active compound of herbal drug in biological samples [11-14]. Consequently, in the present study, a UPLCMS/MS method was established for the determination of dauricine in rat plasma samples and was successfully applied to the pharmacokinetic after oral and intravenous administration in rats. The UPLC-MS/MS method in this study was validated for selectivity, linearity, accuracy, precision, recovery, and stability with a total run time of $3 \mathrm{~min}$.

\section{Experimental}

Chemicals and Reagents. Dauricine (purity, >98\%; Figure 1a) and daurisoline (IS, purity, $>98 \%$; Figure 1b) were purchased from the Chengdu Mansite Pharmaceutical Co. Ltd. (Chengdu, China). Liquid chromatography (LC)-grade acetonitrile and methanol were purchased from Merck Company (Darmstadt, Germany). Ultra-pure water was prepared by Millipore Milli-Q purification system (Bedford, MA, USA). Rat blank plasma samples were supplied by drug-free rats (Laboratory Animal Center of Wenzhou Medical University).

Instrumentation and Conditions. A UPLC-MS/MS system with ACQUITY I-Class UPLC and a XEVO TQD triple quadrupole mass spectrometer (Waters Corp., Milford, MA, USA), equipped with an electrospray ionization (ESI) interface, was used to analyze the compounds. The UPLC system was comprised of a binary solvent manager (BSM) and a sample manager with flow-through needle (SM-FTN). Masslynx 4.1 software (Waters Corp.) was used for data acquisition and instrument control.

Dauricine and daurisoline (IS) were separated using a UPLC BEH C18 column $(2.1 \mathrm{~mm} \times 50 \mathrm{~mm}, 1.7 \mu \mathrm{m}$, Waters, USA) maintained at $40{ }^{\circ} \mathrm{C}$. The initial mobile phase consisted of acetonitrile and water (containing $0.1 \%$ formic acid) with gradient elution at a flow rate of $0.4 \mathrm{~mL} \mathrm{~min}{ }^{-1}$. Elution was in a linear

This is an open-access article distributed under the terms of the Creative Commons Attribution-NonCommercial 4.0 International License (https://creativecommons.org/licenses/by-nc/4.0/), which permits unrestricted use, distribution, and reproduction in any medium for non-commercial purposes, provided the original author and source are credited, a link to the CC License is provided, and changes - if any - are indicated. 
<smiles>COc1cc2c(cc1OC)C(Cc1ccc(O)c(Oc3ccc(C[C@@H]4c5cc(OC)c(OC)cc5CCN4C)cc3)c1)N(C)CC2</smiles><smiles>COc1cc2c(cc1O)[C@H](Cc1ccc(O)c(Oc3ccc(C[C@@H]4c5cc(OC)c(OC)cc5CCN4C)cc3)c1)N(C)CC2</smiles>

(b)

Figure 1. Chemical structure of dauricine (a) and daurisoline (IS, b)

gradient, where the acetonitrile content increased from $30 \%$ to $40 \%$ between 0 and $1.0 \mathrm{~min}$, and then increased to $80 \%$ at 2.0 min. The acetonitrile content was maintained at $80 \%$ for $0.5 \mathrm{~min}$ and then dropped to $30 \%$ within $0.1 \mathrm{~min}$. The total run time of the analytes was $3 \mathrm{~min}$.

Mass spectrometric detection was performed on a triplequadrupole mass spectrometer equipped with an ESI interface in positive mode. Nitrogen was used as the desolvation gas $\left(1000 \mathrm{~L} \mathrm{~h}^{-1}\right)$ and cone gas $\left(50 \mathrm{~L} \mathrm{~h}^{-1}\right)$. Ion monitoring conditions were defined as capillary voltage of $1.5 \mathrm{kV}$, source temperature of $200{ }^{\circ} \mathrm{C}$, and desolvation temperature of $450{ }^{\circ} \mathrm{C}$. Multiple reaction monitoring (MRM) modes of $\mathrm{m} / \mathrm{z} 625.3 \rightarrow$ 206.1 for dauricine and $m / z \quad 611.3 \rightarrow 192.1$ for IS were utilized to conduct quantitative analysis.

Calibration Standards and Quality Control Samples. The stock solutions of dauricine $\left(1.0 \mathrm{mg} \mathrm{mL} \mathrm{mL}^{-1}\right)$ and daurisoline (IS) $\left(1.0 \mathrm{mg} \mathrm{mL}^{-1}\right)$ were prepared in methanol-water (50:50). The $0.5 \mu \mathrm{g} \mathrm{mL}^{-1}$ working standard solution of the IS was prepared from the IS stock solution by dilution with methanol; working solutions for calibration and controls were prepared from stock solutions similarly, using methanol diluent. All of the solutions were stored at $4{ }^{\circ} \mathrm{C}$ and were brought to room temperature before use.

Dauricine calibration standards were prepared by spiking blank rat plasma with appropriate amounts of the working solutions. Calibration plots were offset to range between 3 and $1000 \mathrm{ng}$ $\mathrm{mL}^{-1}$ for dauricine in rat plasma at $2,5,10,20,50,100,200$,
400 , and $600 \mathrm{ng} \mathrm{mL} \mathrm{m}^{-1}$, each by adding $10 \mu \mathrm{L}$ of the appropriate working solution to $100 \mu \mathrm{L}$ of blank rat plasma, followed by short vortex mixing. Quality-control (QC) samples were prepared in the same manner as the calibration standards, in three different plasma concentrations $\left(4,150\right.$, and $500 \mathrm{ng} \mathrm{mL}^{-1}$ ). The calibration standards and QC samples protein were precipitated with acetonitrile before UPLC-MS/MS analysis.

Sample Preparation. Before analysis, the plasma sample was thawed to room temperature. An aliquot of $10 \mu \mathrm{L}$ of the IS working solution $\left(0.5 \mu \mathrm{g} \mathrm{mL}^{-1}\right)$ was added to $100 \mu \mathrm{L}$ of the collected plasma sample in a $1.5-\mathrm{mL}$ centrifuge tube, followed by the addition of $200 \mu \mathrm{L}$ of acetonitrile. The tubes were vortex mixed for $1.0 \mathrm{~min}$. After centrifugation at $14,900 \mathrm{~g}$ for $10 \mathrm{~min}$, the supernatant $(2 \mu \mathrm{L})$ was injected into the UPLCMS/MS system for analysis.

Method Validation. Rigorous tests for selectivity, linearity, accuracy, precision, recovery, and stability, according to the guidelines set by the United States Food and Drug Administration (FDA) and European Medicines Agency (EMA) were conducted in order to thoroughly validate the proposed bioanalytical method [14-24]. Validation runs were conducted on three consecutive days. Each validation run consisted of one set of calibration standards and six replicates of QC plasma samples.

Pharmacokinetic Study. Male Sprague-Dawley rats (200$220 \mathrm{~g}$ ) were obtained from the Laboratory Animal Center of Wenzhou Medical University to study the pharmacokinetics of dauricine. All twelve rats were housed at the Laboratory Animal 
Center of Wenzhou Medical University. All experimental procedures and protocols were reviewed and approved by the Animal Care and Use Committee of Wenzhou Medical University and were in accordance with the Guide for the Care and Use of Laboratory Animals. Diet was prohibited for $12 \mathrm{~h}$ before the experiment, but water was freely available. Blood samples $(0.3 \mathrm{~mL})$ were collected from the tail vein into heparinized $1.5 \mathrm{~mL}$ polythene tubes at $0.0333,0.15,0.5,1,1.5$,

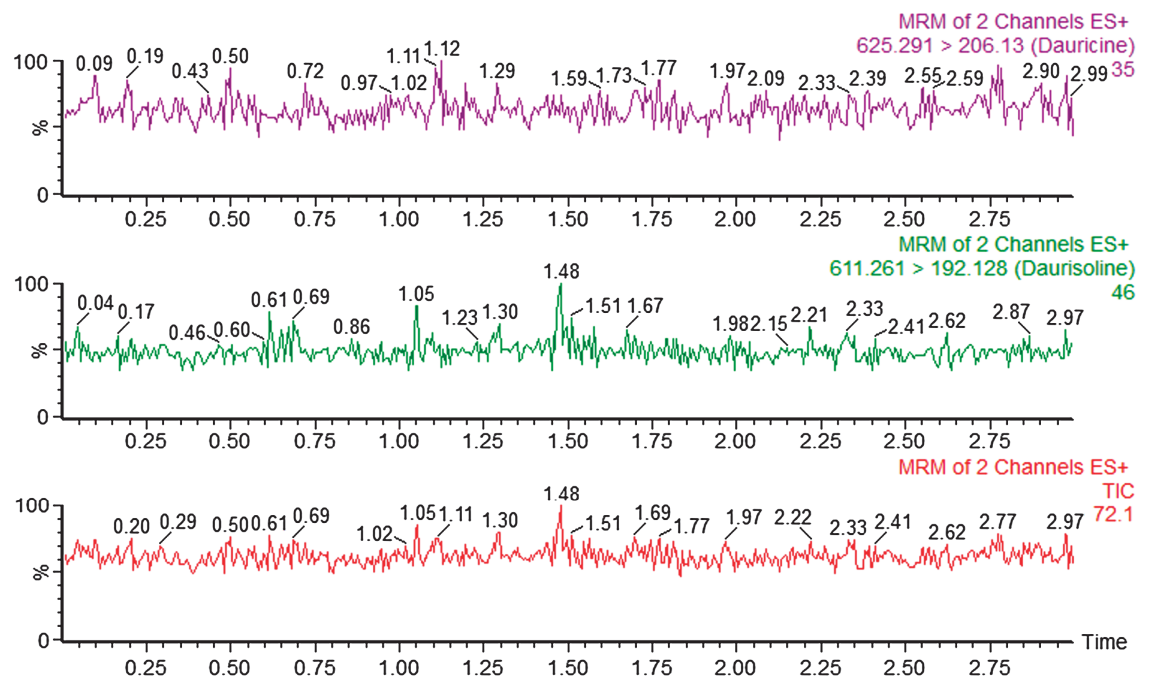

(a)
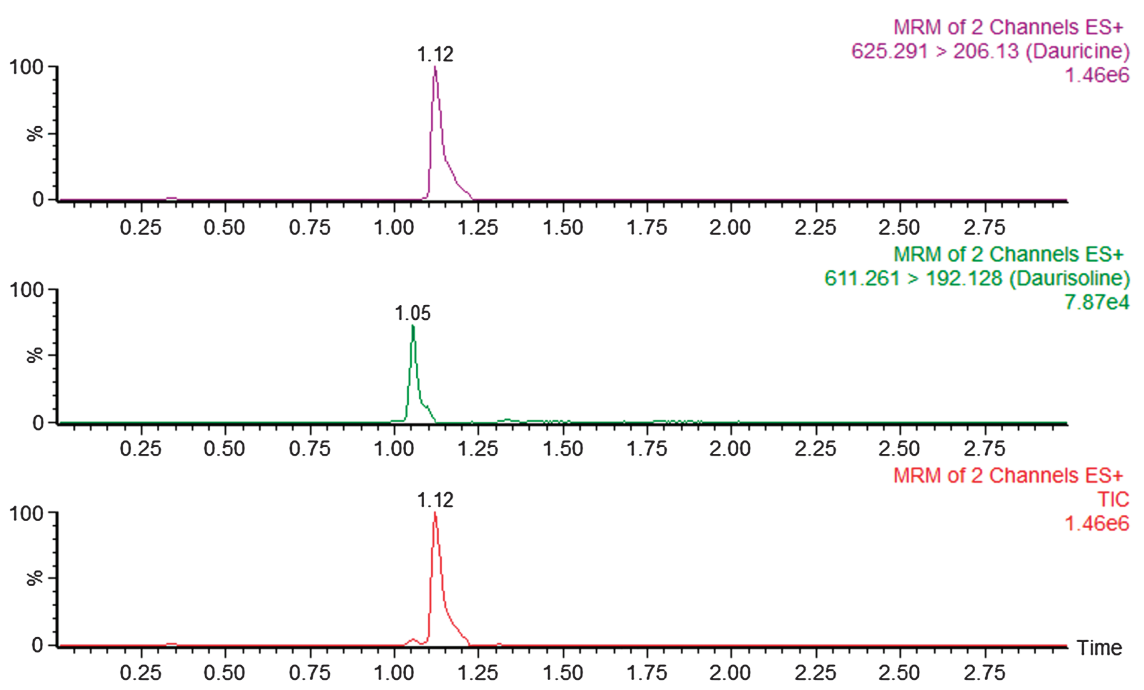

(b)
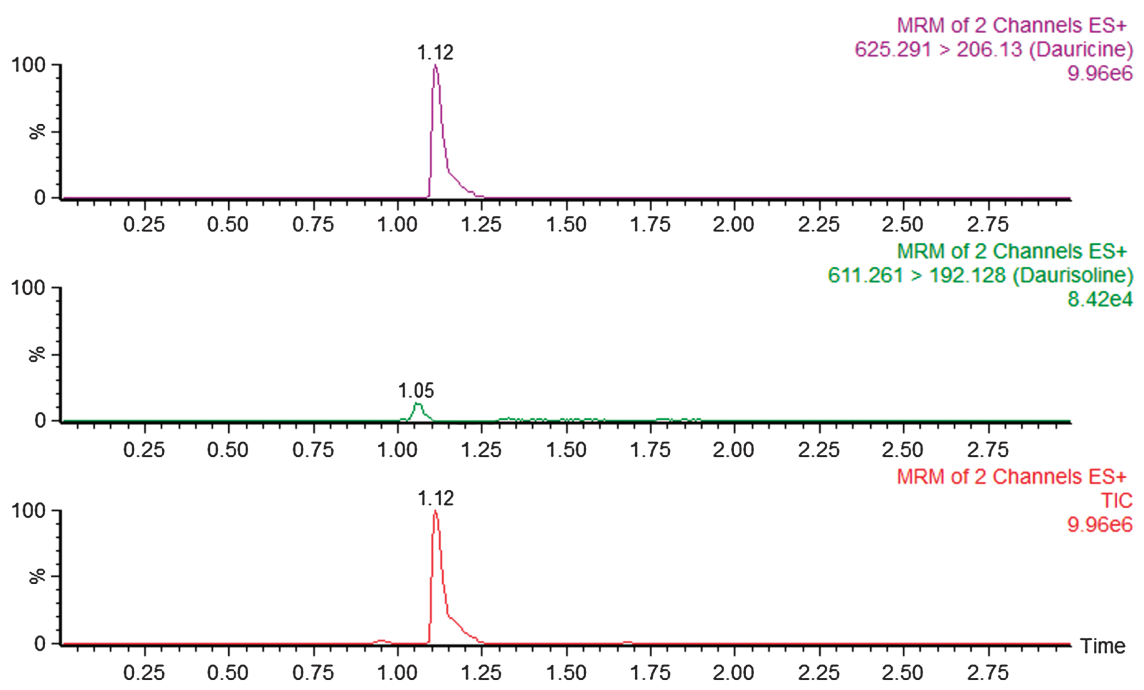

(c)

Figure 2. Representative UPLC-MS/MS chromatograms of dauricine and daurisoline (IS). a, Blank plasma; b, blank plasma spiked with dauricine and IS; c, a rat plasma sample $0.5 \mathrm{~h}$ after intravenous administration of single dosage $2 \mathrm{mg} \mathrm{kg}^{-1}$ dauricine 
and $2 \mathrm{~h}$ after intravenous administration $\left(2 \mathrm{mg} \mathrm{kg}^{-1}\right)$, and at $0.0833,0.15,0.5,1,1.5$, and $2 \mathrm{~h}$ after oral administration $(5 \mathrm{mg}$ $\mathrm{kg}^{-1}$ ) of dauricine. The samples were immediately centrifuged at $3000 \mathrm{~g}$ for $10 \mathrm{~min}$. The plasma as obtained $(100 \mu \mathrm{L})$ was stored at $-20{ }^{\circ} \mathrm{C}$ until analysis.

Plasma dauricine concentration versus time data for each rat was analyzed by DAS (Drug and Statistics) software (version 2.0, Wenzhou Medical University). The maximum plasma concentration $\left(C_{\max }\right)$ was observed directly from the concentration-time curve. The area under the plasma concentrationtime curve (AUC) was estimated by the trapezoidal rule. The plasma clearance (CL), apparent volume of distribution $(V)$, and the half-life $\left(t_{1 / 2}\right)$ were estimated using non-compartmental calculations performed with DAS software.

\section{Results and Discussion}

Selectivity and Matrix Effect. Figure 2 shows typical chromatograms of a blank plasma sample, a blank plasma sample spiked with dauricine and IS, and a plasma sample. There were no interfering endogenous substances observed at the retention time of the dauricine and IS.

To evaluate the matrix effect, blank rat plasma was extracted and spiked with the analyte at 4, 150, and $500 \mathrm{ng}$ $\mathrm{mL}^{-1}$ concentrations $(n=6)$. The corresponding peak areas were then compared to those of neat standard solutions at equivalent concentrations; this peak area ratio is defined as the matrix effect. The matrix effect of the IS was evaluated in a similar manner. The matrix effect for dauricine at concentra-

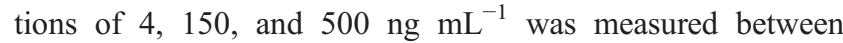
$88.0 \%$ and $90.3 \%(n=6)$ (Table 1$)$. The matrix effect for IS (50 $\left.\mathrm{ng} \mathrm{mL} \mathrm{mL}^{-1}\right)$ was $93.6 \%(n=6)$. As a result, matrix effect from plasma is considered negligible in this method.

Calibration Curve and Sensitivity. Linear regressions of the peak area ratios versus concentrations were fitted over the concentration range of 2-600 $\mathrm{ng} \mathrm{mL} \mathrm{mL}^{-1}$ for dauricine in rat plasma. The equation used to express the calibration curve is the following: $y=(0.000203 \pm 0.0000134) x+(0.000212 \pm$ $0.000032), r=0.9985 \pm 0.0013$, where $y$ represents the ratios of dauricine peak area to that of IS, and $x$ represents the plasma concentration. The lower limit of quantification (LLOQ) was defined as the lowest concentration on the calibration curves. The precision and accuracy at LLOQ should be less than $20 \%$, and accuracy should be between $80 \%$ and $120 \%$. The LLOQ for the determination of dauricine in plasma was $2 \mathrm{ng} \mathrm{mL}^{-1}$. The precision, accuracy, and recovery at LLOQ were $16.4 \%, 90.6 \%$ and $92.2 \%$, respectively. The LOD, defined as a signal/noise ratio of 3, was $0.5 \mathrm{ng} \mathrm{mL}^{-1}$ for dauricine in rat plasma.

Precision, Accuracy, and Recovery. The precision of the method was determined by calculating relative standard deviation (RSD) for QCs at three concentration levels over 3 days of validation tests. Intra-day precision was $13 \%$ or less, and inter-day precision was $11 \%$ or less at each QC level. The accuracy of the method was between $95.8 \%$ and $105.9 \%$ at each QC level. Mean recoveries of dauricine in rat plasma ranged from $91.5 \%$ to $95.1 \%$ (Table 1 ). The recovery of the IS (50 $\mathrm{ng} \mathrm{mL}^{-1}$ ) was $86.4 \%$.

Carry-over. None of the analytes showed any significant peak ( $\geq 20 \%$ of the LLOQ and $5 \%$ of the IS) in blank samples injected after the upper limit of quantification (ULOQ) samples. Adding 0.4 extra minutes to the end of the gradient elution effectively washed the system between samples, thereby eliminating carry-over [25].

Stability. Results from the autosampler showed that the analyte was stable under room temperature, freeze-thaw, and long-term (20 days) conditions, which was confirmed because the bias in concentrations was within $\pm 12 \%$ of their nominal values. To this effect, the established method is suitable for pharmacokinetic study.

Application. The method was applied to a pharmacokinetic study in rats. The mean plasma concentration-time curve after intravenous $\left(2 \mathrm{mg} \mathrm{kg}^{-1}\right)$ and oral $\left(5 \mathrm{mg} \mathrm{kg}^{-1}\right)$ administration of dauricine is shown in Figure 3. Primary pharmacokinetic parameters, based on non-compartment model analysis, are summarized in Table 2.

The bioavailability of dauricine was found to be $55.4 \%$ for the first time.

\section{Conclusion}

In the present study, a simple, precise, and accurate UPLCMS/MS method for the quantitation of dauricine in rat plasma was established, using $100 \mu \mathrm{L}$ of plasma with an LLOQ of $2 \mathrm{ng} \mathrm{mL} \mathrm{m}^{-1}$. The UPLC-MS/MS method was successfully applied to a pharmacokinetic study of dauricine after intravenous

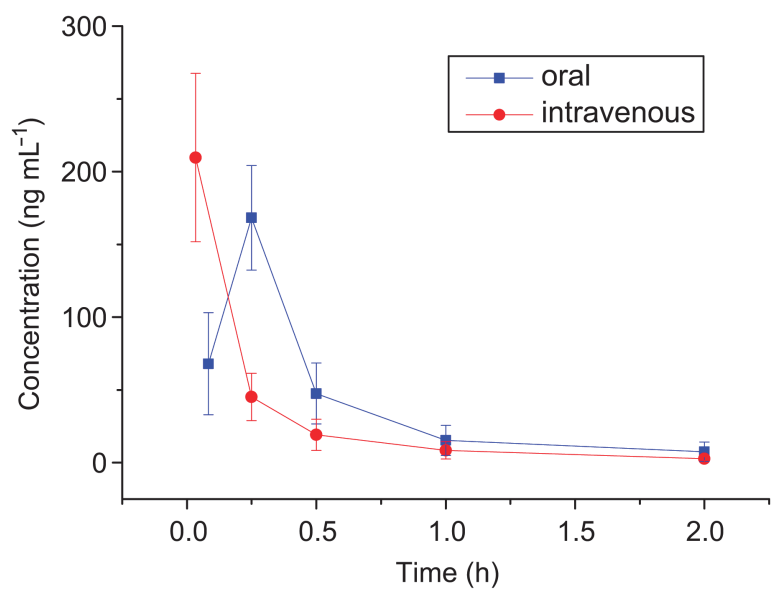

Figure 3. Mean plasma concentration time profile after intravenous $\left(2 \mathrm{mg} \mathrm{kg}^{-1}\right)$ and oral $\left(5 \mathrm{mg} \mathrm{kg}^{-1}\right)$ administration of dauricine in rats

Table 2. Primary pharmacokinetic parameters after intravenous and oral administration of dauricine in rats $(n=6)$

\begin{tabular}{llcc}
\hline Parameters & Unit & i.v. $2 \mathrm{mg} \mathrm{kg}^{-1}$ & p.o. $5 \mathrm{mg} \mathrm{kg}^{-1}$ \\
\hline $\mathrm{AUC}_{(0-t)}$ & $\mathrm{ng} \mathrm{mL}^{-1 * h}$ & $55.3 \pm 15.3$ & $76.7 \pm 21.5$ \\
$\mathrm{AUC}_{(0-\infty)}$ & $\mathrm{ng} \mathrm{mL}$ & $57.7 \pm 55.4$ & $86.4 \pm 29.3$ \\
$\mathrm{MRT}_{(0-t)}$ & $\mathrm{h}$ & $0.3 \pm 0.1$ & $0.5 \pm 0.1$ \\
$\mathrm{MRT}_{(0-\infty)}$ & $\mathrm{h}$ & $0.4 \pm 0.2$ & $0.7 \pm 0.4$ \\
$t_{1 / 2}$ & $\mathrm{~h}$ & $0.5 \pm 0.3$ & $0.7 \pm 0.3$ \\
$\mathrm{CL}$ & $\mathrm{L} \mathrm{h}^{-1} \mathrm{~kg}^{-1}$ & $36.7 \pm 10.1$ & $63.8 \pm 26.3$ \\
$V$ & $\mathrm{~L} \mathrm{~kg}^{-1}$ & $29.5 \pm 22.7$ & $57.9 \pm 12.3$ \\
$C_{\max }$ & $\mathrm{ng} \mathrm{mL}^{-1}$ & $209.7 \pm 57.8$ & $168.4 \pm 35.9$ \\
\hline
\end{tabular}

Table 1. Precision, accuracy, recovery, and matrix effect for dauricine of QC sample in rat plasma $(n=6)$

\begin{tabular}{|c|c|c|c|c|c|c|}
\hline \multirow[t]{2}{*}{ Concentration $\left(\mathrm{ng} \mathrm{mL} \mathrm{m}^{-1}\right)$} & \multicolumn{2}{|c|}{ Precision $(\mathrm{CV} \%)$} & \multicolumn{2}{|c|}{ Accuracy (\%) } & \multirow[t]{2}{*}{ Matrix effect } & \multirow[t]{2}{*}{ Recovery } \\
\hline & Intra-day & Inter-day & Intra-day & $\overline{\text { Inter-day }}$ & & \\
\hline 10 & 12.5 & 8.3 & 102.9 & 96.8 & 90.0 & 92.6 \\
\hline 100 & 7.6 & 10.4 & 104.7 & 95.8 & 90.3 & 95.1 \\
\hline 500 & 10.9 & 7.3 & 99.9 & 105.9 & 88.0 & 91.5 \\
\hline
\end{tabular}


and oral administration. The bioavailability of dauricine was found to be $55.4 \%$.

\section{Disclosure of Conflict of Interest}

The authors declare no conflict of interest.

\section{References}

1 Zhao, J.; Lian, Y.; Lu, C.; Jing, L.; Yuan, H.; Peng, S. J. Ethnopharmacol. 2012, 141, 685-691.

2. Wang, J.; Li, Y.; Zu, X. B.; Chen, M. F.; Qi, L. Asian Pac. J. Trop. Med. 2012, 5, 973-976.

3. Jin, H.; Shen, S.; Chen, X.; Zhong, D.; Zheng, J. Toxicol. Appl. Pharmacol. 2012, 261, 248-254.

4. Yang, Z.; Li, C.; Wang, X.; Zhai, C.; Yi, Z.; Wang, L.; Liu, B.; Du, B.; Wu, H.; Guo, X.; Liu, M.; Li, D.; Luo, J. J. Cell Physiol. 2010, 225, 266-275.

5. Yang, X. Y.; Liu, Q. N.; Zhang, L.; Jiang, S. Q.; Gong, P. L. Am. J Chin. Med. 2010, 38, 307-318.

6. Xie, H.; Liu, Y.; Peng, Y.; Zhao, D.; Zheng, J. Anal. Bioanal. Chem. 2016, 408, 4111-4119.

7. Dong, P. L.; Han, H.; Zhang, T. Y.; Yang, B.; Wang, Q. H.; Eerdun, G W. Mol. Med. Rep. 2014, 9, 985-988.

8. Shi, S. J.; Chen, H.; Gu, S. F.; Zeng, F. D. Acta Pharmacol. Sin. 2003, $24,1011-1015$.

9. Wei, J.; Fang, L.; Liang, X.; Su, D.; Guo, X. Talanta 2015 144, 662-670.

10. Liu, X.; Liu, Q.; Wang, D.; Wang, X.; Zhang, P.; Xu, H.; Zhao, H. Zhao, H. J. Chromatogr. B Analyt. Technol. Biomed. Life Sci. 2010, 878 , 1199-1203.
11. Wen, C.; Wang, S.; Huang, X.; Liu, Z.; Lin, Y.; Yang, S.; Ma, J.; Zhou, Y.; Wang, X. Biomed. Chromatogr. 2015, 29, 1805-1810.

12. Wang, S.; Wu, H.; Geng, P.; Lin, Y.; Liu, Z.; Zhang, L.; Ma, J.; Zhou, Y.; Wang, X.; Wen, C. Biomed. Chromatogr. 2016, 30, 1145-1149.

13. Ma, J.; Wang, S.; Huang, X.; Geng, P.; Wen, C.; Zhou, Y.; Yu, L.; Wang, X. J. Pharm. Biomed. Anal. 2015, 111, 131-137.

14. Wang, X.; Wang, S.; Ma, J.; Ye, T.; Lu, M.; Fan, M.; Deng, M.; Hu, L.; Gao, Z. J. Pharm. Biomed. Anal. 2015, 115, 368-374.

15. Zhang, J.; Geng, P.; Luo, X.; Zhou, G.; Lin, Y.; Zhang, L.; Wang, S.; Wen, C.; Ma, J.; Ding, T. Int. J. Clin. Exp. Med. 2015, 8, 18420-18426.

16. Xu, Y.; Bao, S.; Tian, W.; Wen, C.; Hu, L.; Lin, C. Int. J. Clin. Exp. Med. 2015, 8, 17612-17622.

17. Wen, C.; Zhang, Q.; He, Y.; Deng, M.; Wang, X.; Ma, J. Acto Chromatogr. 2015, 1, 1-11.

18. Tian, W.; Cai, J.; Xu, Y.; Luo, X.; Zhang, J.; Zhang, Z.; Zhang, Q.; Wang, X.; Hu, L.; Lin, G. Int. J. Clin. Exp. Med. 2015, 8, 15164-15172.

19. Wang, S.; Wu, H.; Huang, X.; Geng, P.; Wen, C.; Ma, J.; Zhou, Y.; Wang, X. J. Chromatogr. B Analyt. Technol. Biomed. Life Sci. 2015, 990, 118 124

20. Wang, X.; Wang, S.; Lin, F.; Zhang, Q.; Chen, H.; Wang, X.; Wen, C.; Ma, J.; Hu, L. J. Chromatogr. B Analyt. Technol. Biomed. Life Sci. 2015, 983984, 125-131.

21. Ma, J.; Wang, S.; Zhang, M.; Zhang, Q.; Zhou, Y.; Lin, C.; Lin, G.; Wang, X. Biomed. Chromatogr. 2015, 29, 1203-1212.

22. Zhang, Q.; Wen, C.; Xiang, Z.; Ma, J.; Wang, X. J. Pharm. Biomed. Anal. 2014, 90, 134-138.

23. Ma, J.; Lin, C.; Wen, C.; Xiang, Z; Yang, X.; Wang, X. J. Chromatogr B Analyt. Technol. Biomed. Life Sci. 2014, 953-954, 143-146.

24. Ma, J.; Cai, J.; Lin, G.; Chen, H.; Wang, X.; Wang, X.; Hu, L. J. Chromatogr. B Analyt. Technol. Biomed. Life Sci. 2014, 959, 10-15.

25. Williams, J. S.; Donahue, S. H.; Gao, H.; Brummel, C. L. Bioanalysis 2012, 4, 1025-1037. 